\title{
Utilisation d'un observateur pour le pilotage des systèmes contrôlés par le produit
}

\author{
Olivier Cardin — Pierre Castagna - Etienne Chové \\ Institut de Recherche en Communications et en Cybernétique de Nantes \\ 1 , rue de la Nö̈ \\ F-44321 Nantes cedex 03 \\ \{olivier.cardin, pierre.castagna, etienne.chove\}@irccyn.ec-nantes.fr
}

\begin{abstract}
RÉSUMÉ. Dans un système de production piloté par le produit, le produit devient un acteur intelligent participant à des décisions locales. Pourtant, ce système de production a des objectifs de plus en plus globaux. Nous montrons dans cet article comment la simulation peut être une aide importante pour cette prise de décision locale, en permettant à la fois une vision globale de l'état du système et une prévision prenant en compte le comportement émergeant de l'activité de l'ensemble des centres locaux de prise de décision. Nous présentons un exemple de système piloté par des produits informés où la simulation sert à la fois d'observateur et d'outil d'aide à la décision.

ABSTRACT. In a product-driven system, the product becomes an intelligent actor taking part in local decisions. However, the goal of those systems becomes more and more global. We show in this paper how simulation improves the local decision by allowing a global vision of the state of the system and a prevision of the emerging behavior of the activity of the decisionmaking local centers.

We present an example of system where simulation is used to be an observer of the system and a tool of decision-making support.

MOTS-CLÉs: Pilotage par le produit, observateur, simulation en ligne, aide à la décision.

KEYWORDS: Product-driven, Observer, On-line simulation, decision aid.
\end{abstract}

JESA $-\mathrm{n}^{\circ} \mathrm{x} / 2008$. Systèmes contrôlés par le produit 


\section{Introduction}

\subsection{Emergence du concept de pilotage par le produit}

Nous vivons depuis quelques années une double évolution concernant le pilotage des systèmes industriels, double évolution qui parait de prime abord contradictoire. La première évolution se traduit par une intégration de plus en plus poussée de tous les acteurs du système industriel, depuis les fournisseurs de matières premières jusqu'au service commercial en relation avec le client final. C'est le concept de « supply chain », qui prend la plupart du temps une dimension internationale du fait de la mondialisation. La deuxième évolution, se traduit par une recherche de flexibilité et de dynamisme, d'une part pour répondre à une demande elle-même de plus en plus variée et d'autre part pour mieux réagir aux aléas induits par des systèmes de plus en plus complexes. Cette double évolution conduit à une profonde évolution de la structure des systèmes de production qui passent progressivement d'une organisation hiérarchique, où les décisions sont prises niveau par niveau, du haut vers le bas, chaque niveau communiquant ses décisions aux niveaux inférieurs, à une organisation en réseaux, chaque nœud de ce réseau étant un centre de décision plus ou moins autonome (Trentesaux, 2002). Il est intéressant de constater le caractère fractal de cette évolution. Nous pouvons en effet la constater au niveau le plus global de la « supply chain », celle-ci étant constituée d'un réseau complexe de donneurs d'ordres et de sous-traitants. Mais nous pouvons aussi voir cette évolution au niveau d'un atelier de production, de plus en plus considéré comme un réseau de ressources de production négociant entre elles pour se répartir la charge de travail. $\mathrm{Au}$ plus bas niveau, des capteurs et des actionneurs intelligents deviennent capables de communiquer entre eux à travers des réseaux de terrain. Ce passage d'une structure hiérarchique à une structure hétérarchique se traduit souvent par une importante mutation du principe de pilotage qui de prédictif devient réactif. Le pilotage prédictif est basé sur la notion d'ordonnancement. Ce dernier consiste à prévoir dans le temps la date de réalisation de chaque activité (production, transport, maintenance prédictive, etc.). Ce pilotage est prédictif dans le sens où une décision est prise à un instant donné, mais ne sera appliquée que beaucoup plus tard. L'avantage de cet ordonnancement prédictif réside dans l'approche globale du problème, qui, en traitant l'ensemble des ressources de production, permet de garantir une relative optimisation du comportement du système. Cette optimisation est malheureusement relative à cause de deux problèmes. Le premier réside dans la complexité algorithmique de la plupart des méthodes d'ordonnancement, rendant leurs applications délicates pour des problèmes de taille industrielle. Si l'utilisation de méta-heuristiques permet en partie de résoudre ce premier problème, on se heurte alors à une deuxième difficulté qui est le manque de flexibilité de la solution obtenue. En effet, l'approche globale de l'organisation du système entraine une anticipation des décisions qui ne sont plus pertinentes au moment de leur application. Dans la pratique industrielle, l'efficacité des meilleurs ordonnancements 
est souvent rapidement dégradée à cause des nombreux aléas venant remettre en cause les dates initialement prévues.

Le pilotage réactif est basé sur l'application d'un ensemble de règles de décisions prises en temps réel durant le fonctionnement de l'unité de production. Il ne s'agit pas de supprimer toute prédiction dans le processus de prise de décision, mais de toujours prendre les décisions au plus tard, c'est-à-dire à l'instant de leur application. Cette approche conduit à donner une place plus importante au produit qui, de simple matériau circulant dans le système, devient un acteur du système de pilotage, capable d'interactions intelligentes avec les autres composantes du système. La genèse de cette vision du pilotage se trouve dans le paradigme d'holon (Valckenaers et al., 1998) (Van Brussel et al., 1998). Ainsi s'est développé le concept de pilotage par le produit. Il s'agit de conférer au produit un rôle actif et participatif dans les décisions et les flux d'informations engendrés dans un système de production, pour remplir des objectifs de transformation, de déplacement, de maintenance, de logistique, d'usage et de recyclage (Pétin et al., 2007), (McFarlane et al., 2002).

Ce concept de pilotage est très attrayant. Il permet d'améliorer de façon significative la robustesse de la conduite du système de production, face aux aléas et aux incertitudes de modélisation (Roy, 1989). Pinot et al. (Pinot et al., 2007) ont comparé la robustesse a posteriori des solutions proposées par un algorithme d'ordonnancement prédictif, un algorithme d'ordonnancement de groupes (avec deux niveaux de flexibilité) et une production pilotée par le produit vis-à-vis des temps de transports, non modélisés dans les algorithmes d'ordonnancement.

Si le pilotage par le produit répond à un besoin d'évolution des structures de pilotage vers plus de flexibilité, il a été rendu possible par l'émergence des techniques d'identification automatique RFID (Radio Frequency Identification), conférant au produit des facultés de communication et de stockage d'information (Cea et Bajic, 2004).

\subsection{Problématiques}

L'évolution d'un pilotage prédictif basé un la notion d'ordonnancement vers un pilotage réactif basé sur des prises de décision en temps réel n'est pas sans poser des problèmes que nous aborderons dans cet article.

Le premier concerne la définition même de la notion de pilotage. Qu'est-ce qu'un système piloté par le produit? Que deviennent les fonctions de base assurées par le pilotage dans un système contrôlé par le produit? À travers la section 2 nous indiquerons notre vision du pilotage dans le contexte d'un système contrôlé par le produit. 
Le deuxième problème concerne la qualité du pilotage. À travers celui-ci, nous cherchons un fonctionnement de la production qui soit le meilleur possible, c'est-àdire un fonctionnement qui optimise un ou plusieurs critères globaux. Dans un fonctionnement prédictif, cette optimisation globale est à la base même du pilotage. L'ordonnancement cherche à placer les tâches en optimisant un critère (par exemple le makespan pour durée totale d'exécution des tâches). Dans un système contrôlé par le produit, le comportement global émerge de l'ensemble des décisions prises en temps réel. Il n'est donc pas connu à l'avance et toute tentative d'optimisation semble bien difficile. Nous montrerons, dans la section 3 comment la simulation en ligne, sans aller jusqu'à permettre une optimisation globale du comportement, va améliorer la prise de décision locale en permettant une meilleure prévision des conséquences de la décision. Les premiers résultats nous amènent à penser que le pilotage par le produit ne peut être pensé sans un outil d'aide à la décision permettant de prédire sur un terme relativement court le comportement de la production.

Le troisième problème concerne la vision du système prise en compte dans le pilotage. Dans un ordonnancement prédictif, c'est l'ensemble de l'unité de production qui est prise en compte dans la construction de l'ordonnancement des tâches. Les décisions prises dans un SCP sont souvent locales, dans le sens où elles agissent localement sur une petite partie du système et surtout dans le sens où elles ne sont prises qu'en ayant une vision très restreinte du système. Nous montrerons dans cette même section 3 comment un observateur basé sur la simulation temps réel nous permet d'élargir notre vision du système pour une meilleure prise de décision locale.

La dernière partie nous permettra de présenter l'application de ces concepts à un système de production ayant une taille réelle. Ce système est intéressant dans le sens où une partie du pilotage est informé par le produit, celui-ci déclenchant les décisions qui le concernent à l'instant de l'application de la décision. Une autre partie du pilotage est réalisée de façon plus hiérarchique à travers les décisions d'un pilote de ligne. La structure de pilotage proposée se trouve entre une structure purement isoarchique et une structure complètement hiérarchique. Nous montrons que pour ces deux couches du pilotage, la simulation permet une aide à la décision.

\section{Prise de décision dans un contexte de pilotage par le produit}

\subsection{La fonction pilotage dans le contexte du contrôle par le produit}

Dans le contexte des systèmes contrôlés par le produit, (Gouyon et al., 2007) proposent une architecture de pilotage basée sur le concept de système de production holonique.

Ce système est distribué, basé sur des coopérations entre des contrôleurs de ressource assurant la bonne exécution des transports et des opérations de production 
et des contrôleurs de produit assurant que les produits subissent la succession des opérations permettant leur fabrication. Gouyon et al. proposent d'utiliser la reconfiguration dynamique de la commande introduite par (Brennan et al. 2002).

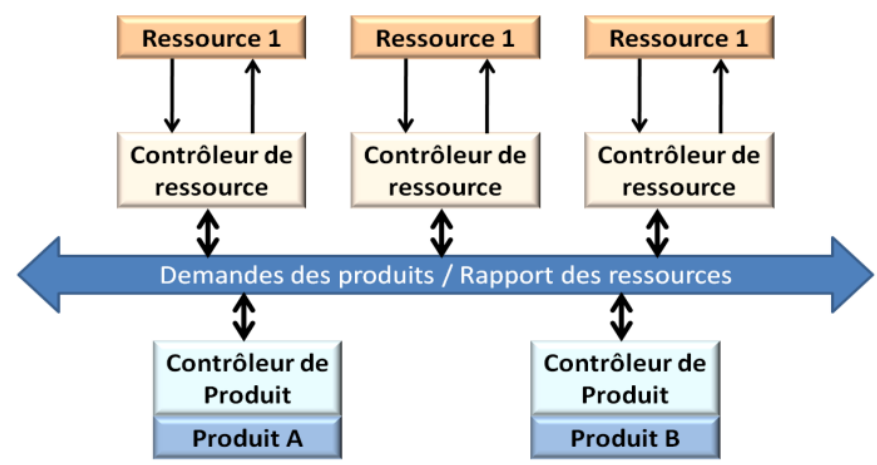

Figure 1. Architecture de pilotage contrôlé par le produit (Gouyon et al., 2007)

Dans ses travaux concernant la mise en place d'un environnement pour l'évaluation des systèmes contrôlés par le produit, R. Pannequin propose aussi un pilotage distribué, implémenté sur une plateforme multi-agent (Pannequin, 2007).

De façon très générale, (Pujo et Kieffer, 2002) définissent le pilotage de la production comme étant l'ensemble des fonctions permettant le lancement et le suivi de la production, c'est-à-dire le contrôle en temps-réel des ordres de fabrication planifiés, qui contiennent, entre autres, le triplet \{tâche ; ressource ; date).

Dans le contexte du pilotage par le produit, nous proposons d'ajouter à cette définition l'ensemble des décisions induites par les flux de produits, permettant un fonctionnement acceptable du système de production par rapport à un ou plusieurs critères de performance. En effet, ce qui nous semble caractériser le pilotage des SCP sont ces décisions, provoquées par le flux de produits. Cette définition implique que deux tâches doivent être réalisées conjointement: un suivi de la production, contenant notamment une remontée d'informations depuis le système de production, et un ensemble de prises de décision, s'appuyant notamment sur les données précédentes.

La notion de décision étant au centre du pilotage par le produit, il nous parait important de préciser ici le cadre d'une prise de décision. Chaque décision peut être décomposée en 5 caractéristiques:

1. Le Déclencheur de la décision (D): évènement déclenchant la prise de décision. 
2. Le Centre de décision (C): entité intelligente du système évaluant la décision.

3. Les Paramètres de la décision (P): Ensemble des données, mesurées, évaluées ou prévues qui influent sur la prise de décision.

4. Le Système Directement impacté par la décision $\left(\mathrm{S}_{\mathrm{d}}\right)$ : Sous-ensemble du système sur lequel la décision a un impact direct.

5. Le Système Influé par la décision $\left(\mathrm{S}_{\mathrm{i}}\right)$ : sous-ensemble du système sur lequel la décision a une influence induite.

Si l'on prend l'exemple du pilotage d'un atelier en flow-shop représenté Figure 2: après le passage sur la machine 1 , une décision doit être prise pour le passage sur les machines 2 ou 3 . Le déclencheur de la décision correspond alors à la disponibilité du produit en sortie de machine 1, le centre de décision se situe au niveau de la partie commande de la machine 1. Les paramètres de la décision sont à la fois les relevés de niveaux de stock des machines 2 et 3 , se trouvant dans une base de données mise à jour en temps réel par les centres de décision des machines 2 et 3 , ainsi que les informations concernant le produit en sortie de la machine 1 ( priorité, durée opératoire, due date, ...) portées par le produit. Le système directement influé par la décision est, outre le produit, la machine choisie et en priorité son stock, alors que l'autre machine constitue ad minima le système influé par la décision, puisqu'elle n'aura pas à traiter le produit.

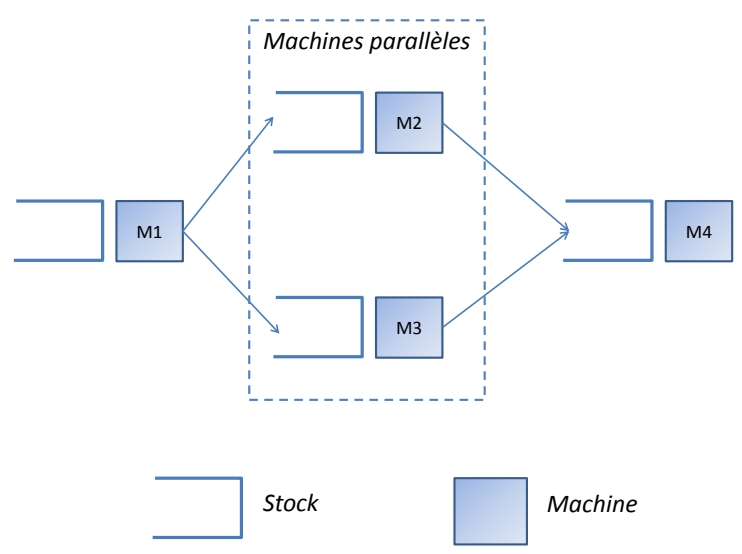

Figure 2. Flow shop à machines parallèles

Ces définitions nous amènent à préciser ce que nous entendons par «systèmes contrôlés par le produit ». Nous utilisons le concept de produit intelligent (Wong et al. 02), qui est défini comme un objet dual physique et informationnel, doté de capacités de mémorisation, de communication, d'action, de décision qui lui permettent de : 
Utilisation d'un observateur pour le pilotage des systèmes contrôlés par le produit 7

1. Posséder une identification unique;

2. Communiquer avec son environnement;

3. Mémoriser et gérer des informations qui lui sont propres;

4. Disposer d'un langage de dialogue et d'échange de ses informations et états;

5. Participer aux processus de décision durant son évolution;

A partir de ces fonctions de base, Wong et al. proposent deux niveaux d'intelligence du produit :

1. A ce niveau, le déclencheur de la décision (D) est l'évènement correspondant à l'arrivée d'un produit en sortie de la machine M1. De même le produit contient tout ou partie des paramètres $(\mathrm{P})$ de la décision. Par contre, le centre de décision est une entité du système de production extérieure au produit. Ce niveau couvre tout autant les produits possédant une identification simple (code-barres, etc.) que les produits captant euxmêmes de l'information (produits instrumentés) ou encore les produits possédant une capacité de stockage en lecture/écriture (type étiquette électronique). Ce niveau intègre les fonctions 1 à 3 du produit intelligent défini par Wong et al., qui parlent alors de «produit orienté information ».

2. Au niveau 2, le produit est à la fois l'auteur de l'évènement déclencheur et le centre de la décision. C'est lui qui évalue la décision sur la base de paramètres, qu'il peut éventuellement contenir ou recueillir sur le système réel. Ses facultés de communication lui permettent éventuellement de communiquer avec d'autres centres de décisions afin de faire ses choix. $\mathrm{Ce}$ niveau intègre les produits capables à la fois d'évaluer la pertinence des solutions possibles et d'interagir avec le système pour l'application de la décision. Ce niveau intègre l'ensemble des fonctions du produit intelligent définies par Wong et al.

Comme on peut le voir dans ces définitions, la nature du produit et la relation entre le produit et son environnement sont prépondérants dans la classification.

L'application qui sera présentée au chapitre 4 fera appel à des produits de niveau 1 dans la métrique proposée par Wong et al.

\subsection{Structure d'un produit intelligent.}

La mise en place d'un SCP impose d'avoir un produit pour le moins informé voire intelligent, ce qui n'est pas sans poser un problème économique, notamment dans les systèmes à haute cadence caractérisés par des flux très importants de produits ayant une faible valeur. Lorsque les encours de production sont relativement faibles, une solution consiste à augmenter le produit en l'associant à un composant qui lui apportera l'intelligence. Ainsi, pour des raisons à la fois économiques et d'interopérabilité des moyens, le produit intelligent, dans un 
système de production, est souvent la combinaison de plusieurs composants (Figure $3)$.

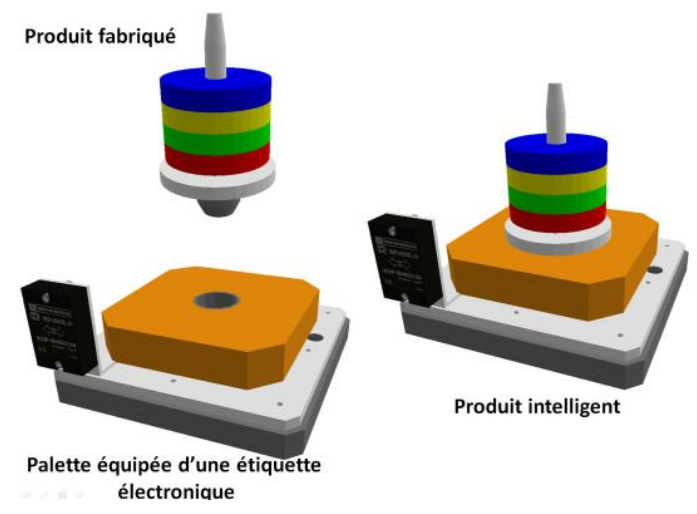

Figure 3. Structure d'un produit intelligent

La structure la plus simple est constituée du produit en cours de fabrication luimême et d'une base associée au produit, comme par exemple une palette transportant ce produit. C'est l'ensemble produit + base qui constitue le produit orienté information. De façon plus générale, la structure du produit intelligent peut être plus complexe. Considérons un chariot automatisé transportant un bac de produit. L'ensemble forme un produit intelligent de niveau 2. Le bac transporté, luimême équipé d'une étiquette électronique et d'un système de décision est aussi un produit intelligent de niveau 2. Les produits transportés, équipés de tags RFID sont eux-mêmes un ensemble de produits intelligents de niveau 1.

Cette vision du produit intelligent met en évidence des opérations importantes d'association et de dissociation, au cours de la production. La dépose d'un produit sur une palette est une opération d'association conduisant à la définition d'un nouveau produit intelligent. Le démontage du produit de cette palette et une opération de dissociation. Un échange d'informations entre le produit et la base s'effectue généralement aux moments de l'association et de la dissociation. Notons que l'association de plusieurs produits intelligents de niveau 1 ou 2 peut poser des problèmes de positionnement et de redondance de l'information. L'association de plusieurs produits intelligents de niveau 2 peut introduire des problèmes de compatibilité de prises de décision, plusieurs centres de décisions se trouvant intégrés dans le produit intelligent final.

Le pilotage d'un SCP est donc caractérisé par une architecture distribuée du pilotage, chaque ressource étant contrôlée par un centre de décision. D'autre part, un ensemble de décisions prises par ces contrôleurs de ressources et de produits sont 
naturellement disséminées au sein d'un SCP. La répartition de l'intelligence permet la simplification du pilotage. Par contre, la prise de décision dans de tels systèmes peut être simpliste, car les paramètres mis à disposition de chaque centre de décision ne concernent généralement qu'un sous-ensemble du système de production (généralement le voisinage du $S_{d}$ ), alors que l'impact de la décision sur le $S_{i}$, voire sur l'ensemble du système, peut être très significatif. L'évaluation de la meilleure décision nécessite souvent:

1. Une faculté de vision augmentée du système pour avoir à disposition un espace $\mathrm{P}$ plus grand;

2. Une faculté de prévision lui permettant d'anticiper les effets sur le $\mathrm{S}_{\mathrm{i}}$.

Comme montré dans (Valckenaers et al., 2006), l'efficacité d'une architecture holonique repose sur la capacité des holons à prévoir le comportement futur du système. Nous pensons que ceci reste particulièrement vrai dans le cas des SCP. Nous proposons dans la suite d'utiliser les concepts définis par (Cardin, 2007) à propos de l'utilisation de la simulation de flux en ligne pour l'aide au pilotage des systèmes contrôlés par le produit.

\section{Apport de la simulation dans la prise de décision}

Dans le contexte défini précédemment, la simulation peut à notre sens intervenir à deux niveaux dans la prise de décision:

1. La simulation fait partie intégrante des méthodes de calcul utilisées par le centre de décision pour l'évaluation de la décision, ce qui correspond aux facultés de prévision;

2. La simulation est utilisée par le centre de décision comme aide à l'obtention de paramètres non-mesurables directement sur le système, ce qui correspond aux facultés de vision.

\subsection{Prévisions de comportement}

Nous proposons dans cette section l'utilisation d'un outil d'aide à la décision basé sur la simulation à évènements discrets. À la fin des années 1990, plusieurs réflexions ont été menées sur l'avenir de la simulation (Drake et Smith, 1996) (Davis, 1998). L'idée motrice de ces réflexions a été de passer d'une simulation destinée à la conception d'un système de production à une simulation pour l'aide au pilotage. Ce passage a bien évidemment d'abord été envisagé pour les grandes installations, où des modèles de simulation avaient déjà été construits. Dans (Castagna et al., 2001), les auteurs répondent à une demande de l'industrie aéronautique qui voulait un simulateur permettant d'avoir une prévision claire du comportement de leur unité de production sur un horizon court (une journée). Le 
concept associé a notamment été exposé dans (Davis, 1998) sous le nom de simulation en ligne.

Le comportement local des systèmes contrôlés par le produit est généralement facile à exprimer par simulation, étant donné qu'il n'est constitué que d'un ensemble de règles locales que chaque entité respecte. Par exemple, le choix d'un produit à faire passer sur une machine au sein de son stock amont se fait selon un critère tel FIFO ou Earliest Due Date cherchant à optimiser localement un critère de performance de la machine concernée, et non en fonction d'un ordonnancement ayant pris en compte l'intérêt du système global.

Il est à l'opposé relativement difficile de connaître le comportement global, émergeant des comportements de chaque entité et de l'impact des décisions prises sur l'ensemble du système. La simulation est aujourd'hui le seul outil permettant la prévision de ce comportement émergent quelle que soit la complexité du système. Le principe de l'utilisation de la simulation est de répondre à des questions de type «Et si? ». Ainsi, chaque centre de décision du système peut, en théorie, faire appel à une ou plusieurs simulations en ligne dans le but de prévoir l'incidence de chacune des alternatives envisagées sur le comportement global du système, et donc notamment sur celui du $\mathrm{S}_{\mathrm{i}}$. En fonction du niveau de contrôle par le produit, l'appel à la simulation se fera éventuellement différemment:

1. Dans un niveau 1 , les centres de décision sont intégrés au système de commande. La simulation en ligne peut alors être envisagée comme une entité de ce système de commande, partie du MES par exemple.

2. Au sein d'un niveau 2 , le produit pilotant peut accéder à des résultats de simulation, soit en instanciant des outils présents au sein du système, soit en utilisant ses propres capacités de calcul pour réaliser ses propres simulations.

$\mathrm{Au}$ vu de la complexité de mise en œuvre de la seconde solution, il est toutefois probable que la première soit généralement préférée: l'outil de simulation est alors inséré dans le système de commande du système, quel que soit le niveau de contrôle par le produit du système.

L'utilisation de la simulation en ligne nécessite un modèle de simulation qui, compte tenu des délais très courts pour prendre la décision, doit utiliser des modèles déjà existants. En effet, comme expliqué dans (Cardin, 2007), les temps de réaction demandés à une telle étude sont trop courts pour envisager la programmation d'un nouveau modèle. Seul le paramétrage des modèles se fait en ligne. Une bibliothèque de modèles est donc à la disposition de l'outil au cours de la production: le centre de décision choisit dans cette bibliothèque le modèle le plus adapté à l'étude. Au vu des objectifs de prévision globale, il semble alors judicieux de centraliser les modèles dans une bibliothèque centrale accessible par tous, et de distribuer les moteurs de simulation au plus près des centres de décision locaux. 
Ainsi implantée, la simulation permet de fournir au centre demandeur l'évolution globale du système à partir d'un état parfaitement connu dans le cas de l'application d'une alternative de la décision. Cette simulation en ligne n'est pas sans poser quelques problèmes. Le premier consiste à démarrer cette simulation à partir d'un état non-vide du système. La plupart des outils de simulation, développés pour concevoir des systèmes complexes ne permettent pas cette initialisation dans un état quelconque. L'ouverture de plus en plus grande des outils de simulation comme QUEST, ARENA ou WITNESS permettent aujourd'hui de résoudre en grande partie ce premier problème.

Le deuxième problème est la connaissance de l'état initial de la simulation. Comme pour toute simulation en ligne, cet état initial se doit d'être fidèle à l'état du système de production au moment de la demande de simulation. Or, la nature généralement distribuée des systèmes contrôlés par le produit rend cette vision difficile. Ce problème d'état initial rejoint donc le problème de vision décrit précédemment, avec la contrainte de la possibilité de traitement informatique, pour pouvoir intégrer très rapidement les données issues de l'état du système dans la simulation.

\subsection{Avoir une vision globale sur le système}

Notre objectif a donc été de déterminer un moyen efficace d'étendre la vision que peut avoir un pilote sur un système contrôlé par le produit. Du fait du caractère pouvant être chaotique de cette classe de systèmes, dans lesquels une simple modification du comportement local d'un élément peut avoir de grandes conséquences sur le comportement émergent du système complet, il nous semble nécessaire d'obtenir une précision importante concernant les données constituant l'état du système. Nous avons choisi d'examiner trois principales possibilités pour avoir une vision complète et à jour de l'état du système à tout instant. Les paragraphes suivants détaillent ces possibilités. Leurs conclusions traitent des conditions d'applicabilité de chacune des solutions.

\subsubsection{Données directement collectées sur le système réel}

La première solution que nous avons envisagée, pour connaître l'état global du système, est de collecter des données issues du système réel, au travers de la commande, du MES ou des composantes supérieures de la décomposition CIM, telles que l'ERP ou l'APS (Figure 4), à condition que les informations collectées soit mises à jour avec une période suffisante.

Cette solution a plusieurs avantages. Les données récupérées sont fiables et représentent l'état réel du système de production. 


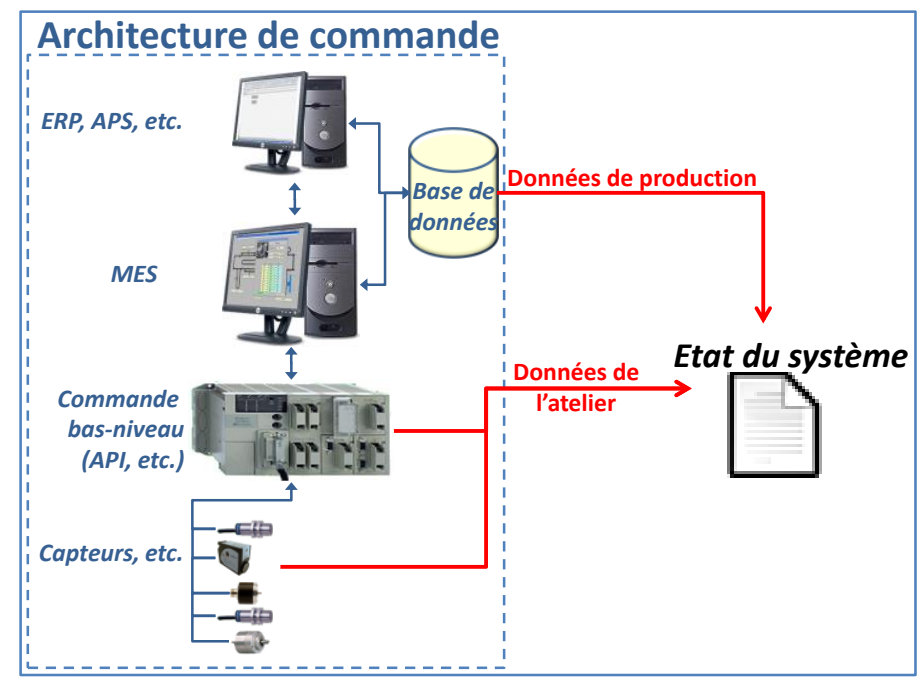

Figure 4. Données directement collectées sur le système de production

La composante la plus importante de cette collecte de données est la performance des capteurs et détecteurs installés sur le système. Certains capteurs pourront nous renseigner en continu sur l'évolution d'une grandeur du système. Un capteur de température pourra par exemple nous donner en continu les conditions à l'intérieur d'un four. Toutefois, en général, beaucoup de données ne sont pas mesurées de cette manière. Prenons l'exemple de la position de véhicules autoguidés (AGV) dans un atelier. Des détecteurs disposés à certains points du circuit (intersections, postes de travail, etc.) suffisent pour piloter la flotte. Le problème est qu'une grande incertitude nait de l'utilisation de tels dispositifs. En effet, lorsqu'un AGV est en face d'un détecteur, on connaît à la fois sa position et la date courante. Dès qu'il se déplace entre deux détecteurs, sa position est totalement inconnue (exceptée l'information disant qu'il se situe sur le tronçon entre les deux détecteurs), et sa date de passage devant le prochain détecteur est indéterminée. Il est impossible que l'ensemble de la flotte d'AGV ne se situe en face des détecteurs présents sur le système à chaque moment où un centre de décision local a besoin des informations. De ce fait, le MES n'a accès, à chaque instant, qu'à une vue partielle de l'état complet de l'atelier : c'est ce que l'on a pu définir comme étant les incertitudes spatiales et temporelles du MES (Cardin et Castagna, 2006).

Nous avons de plus pris le parti dans ces travaux de n'utiliser que les capteurs et détecteurs strictement utilisés par la commande. Pour reprendre l'exemple précédent, l'utilisation des technologies telles qu'un module GPS ou un système de reconnaissance d'image serait envisageable pour obtenir la position exacte des $\mathrm{AGV}$, mais peut également s'avérer très coûteuse, d'autant qu'il sera souvent 
indispensable de coupler plusieurs de ces technologies pour obtenir l'ensemble des informations nécessaires à la définition de l'état du système. Cette contrainte permet de limiter les coûts d'installation de notre système de perception globale du système.

La conclusion que nous avons tirée est que l'utilisation de cette solution est limitée, du fait du manque de disponibilité de certaines des données, prépondérantes dans la définition de l'état du système. Toutefois, elle est à conseiller dans les rares cas particuliers permettant son utilisation principalement grâce à la fiabilité des données obtenues et à la simplicité de mise en œuvre de la solution. Nous citerons par exemple le contrôle aérien où la position, le plan de vol et la trajectoire actuelle de chaque appareil sont parfaitement connus à chaque instant (Rogers et Flanagan, 1991).

\subsubsection{Utilisation d'un simulateur temps-réel}

Depuis quelques années, la plupart des éditeurs de logiciels de simulation proposent des versions dites «temps-réel » de leurs outils. Ces simulateurs ont la particularité de ne pas tourner à la vitesse maximale admise par l'équipement informatique concerné mais de caler leur horloge sur le temps du système réel (souvent appelé wall clock), d'où le nom de simulateur temps-réel. Ces simulateurs ont été développés pour répondre à des besoins d'utilisation de la simulation en temps qu'émulateur de système physique. L'idée initiale est de réduire la durée de mise au point de systèmes complexes de production en réalisant les tests du système de commande en le couplant sur cet émulateur, sans attendre l'existence du système physique.

Notre idée est d'utiliser un tel simulateur, s'exécutant parallèlement à la production. Ce simulateur réagit aux sollicitations de production venant du MES ou de l'ERP, tout comme le système de production réel. Ainsi, il permet de créer une « image » permanente du système réel, que nous considérons comme étant la plus complète approximation de l'état réel du système (Figure 5). Cette solution offre deux avantages importants par rapport à l'utilisation directe des informations issues $\mathrm{du}$ système réel. Le premier est qu'elle supprime le problème des incertitudes spatiales et temporelles définies précédemment. Si nous revenons un instant sur l'exemple de l'AGV précédemment présenté, le simulateur permet de connaître sa position actuelle à tout temps. 


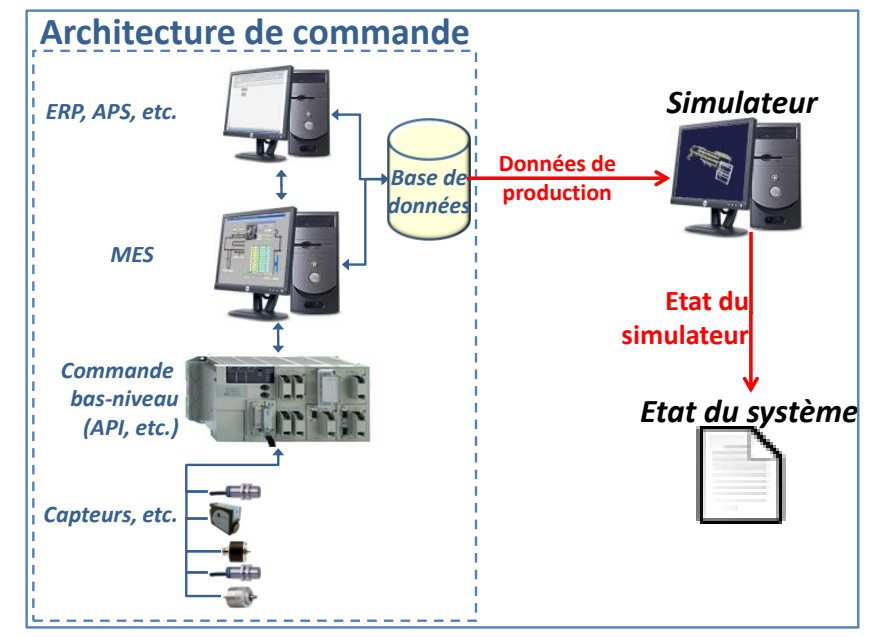

Figure 5. Utilisation d'un simulateur temps-réel

Un second avantage est que l'état récupéré avec cette méthode est très bien formaté pour un traitement informatisé ultérieur. Un tel traitement informatisé est généralement nécessaire tant l'état récupéré est fourni et complexe.

Plusieurs problèmes se posent néanmoins lors de l'application de cette solution. Tout d'abord, l'initialisation de ce simulateur : celui-ci doit démarrer sur un état connu du système de production, qui sera typiquement choisi « vide et inactif » (empty and idle), car c'est généralement l'état le plus facile à décrire complètement «à la main ». De plus, les défauts dans la modélisation du système et les différents aléas qui surviennent au cours de toute production entraînent une déviation sur le résultat final. L'aspect additif de ces écarts au cours du temps finirait par avoir un effet qui peut être loin d'être négligeable.

Pour répondre à cela, plusieurs contraintes doivent être imposées pour garantir l'applicabilité de la solution. Tout d'abord, le simulateur doit être extrêmement fidèle pour limiter au maximum les erreurs dues à la modélisation. Ensuite, il sera préférable de considérer des systèmes qui reviennent régulièrement à un état connu (généralement vide). On pourra citer par exemple des systèmes de production qui se vident chaque jour en fin de journée. On parle de systèmes à cycles régénératifs. Ceci permettra à la simulation d'être réinitialisée, et donc de rattraper les erreurs qui auront été commises. Enfin, il est préférable que le système considéré ait une fréquence d'occurrence d'aléas faible relativement à l'horizon de simulation sans remise à jour. En effet, ces aléas ne peuvent être directement pris en compte dans la modélisation du simulateur et ajoutent donc directement à la déviation du simulateur. 
La classe de systèmes qui peut être considérée est donc restreinte, mais cette solution a l'avantage de constituer une alternative possible et crédible pour des applications de taille limitée qui ne peuvent se permettre les investissements nécessaires à l'utilisation des autres solutions et qui ne nécessitent pas l'obtention d'une précision importante.

\subsubsection{Utilisation d'un observateur}

Comme nous avons pu le voir dans les paragraphes précédents, les deux solutions que nous avons initialement envisagées se sont avérées bien adaptées pour des classes restreintes d'application. Ainsi, pour collecter les données du système en temps réel, il faut que celles-ci soient disponibles avec une période de rafraichissement suffisante. De même, l'utilisation d'un simulateur temps réel suppose que le système passe régulièrement par un état connu, vide par exemple, pour supprimer les dérives accumulées. Dans un souci de construire une solution applicable à la classe de systèmes la plus élargie possible, nous avons donc eu l'idée d'hybrider les deux solutions proposées précédemment, comme présenté dans la Figure 6. Ainsi, nous pensons gagner les avantages du simulateur temps-réel au niveau de la disponibilité des données et ceux de l'utilisation directe des données du système réel au niveau de la fiabilité des données.

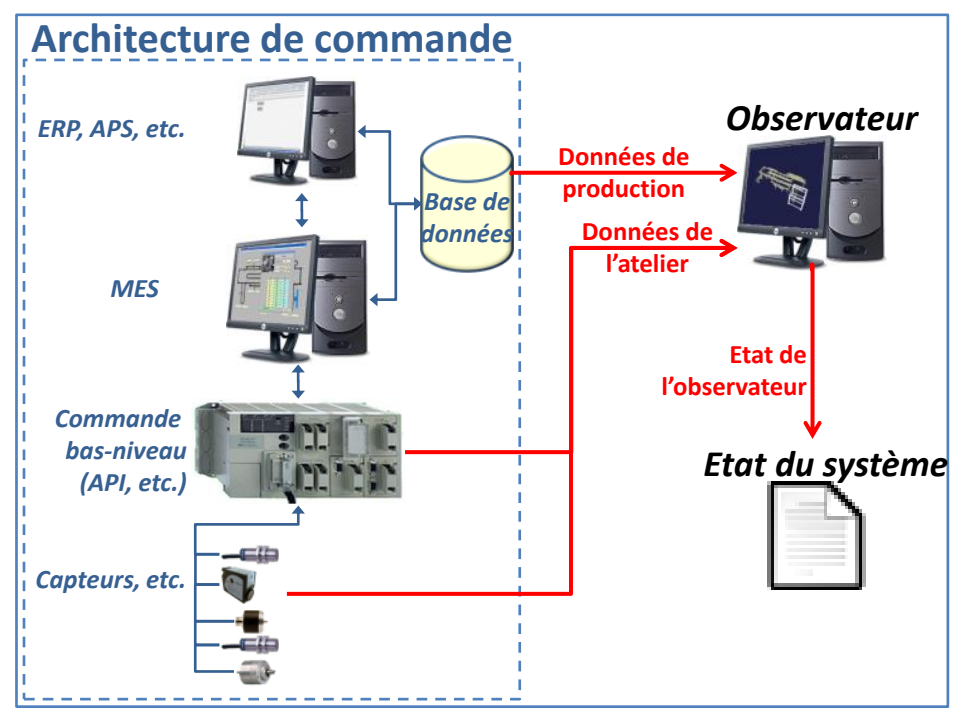

Figure 6. Utilisation d'un observateur

La Figure 6 présente les principes d'une telle solution. Par rapport à la solution précédente, le simulateur est complété par une couche d'observation en temps réel qui gère les données en provenance de la commande bas-niveau. L'ensemble 
(simulateur temps-réel et couche d'observation) constitue un observateur du système de production. En utilisant les données collectées par la couche d'observation, cet ensemble est capable de recaler ses prévisions sur ce qui s'est effectivement passé sur le système. Ce recalage permet d'éviter toute dérive de l'état du simulateur temps-réel par rapport à la réalité. De ce fait, cet observateur tournant à la même vitesse que le système réel, il constitue une image très complète du système. Ainsi, pour connaitre l'état du système réel, le décideur local peut s'adresser directement à cet observateur.

Nous pensons que cette solution est la plus performante implémentable avec les contraintes exprimées précédemment. Mais, même si les coûts d'implantation sont relativement réduits, cette solution reste complexe à mettre en œuvre et est donc réservée aux grandes installations. Lorsque l'on traite de productions contrôlées par le produit, ces contraintes sont généralement respectées car les installations sont rapidement de taille assez importante.

\section{Application a une ligne d'assemblage}

Nous avons appliqué les concepts énoncés précédemment au pilotage d'une ligne d'assemblage. Cette ligne a un double avantage : tout d'abord permettre d'appliquer les concepts développés sur un système physique «taille réelle » muni de son système d'informations (en l'occurrence un MES) et piloté par une commande de type industrielle, constituée d'un ensemble de quatre automates (API) connectés en réseau et d'un PC assurant la supervision. L'ensemble est donc piloté par cinq centres de décision. Ensuite, la nature relativement déterministe du système permet une approche expérimentale efficace. Par exemple, le système nous permet de comparer les résultats obtenus par application de différentes politiques de pilotage.

\subsection{La ligne d'assemblage}

Le système de production étudié (Figure 7) est situé au département Qualité, Logistique Industrielle et Organisation (QLIO) de l'Institut Universitaire de Technologie (IUT) de Nantes. Il a été conjointement financé par l'IUT et L'Atelier Inter-établissement de Productique (AIP PRIMECA) des Pays-de-Loire, puis développé au sein du département QLIO. Ce système, une ligne d'assemblage automatisée, fait partie d'un ensemble de production plus large, comprenant un magasin dynamique automatisé, et un ensemble de transport comportant deux chariots optoguidés. La finalité de cet ensemble est à la fois la formation des étudiants de l'IUT et la recherche.

Ce système, de type job shop avec transferts automatisés, est composé de six postes de travail. Les produits sont transportés par 42 palettes se déplaçant sur des convoyeurs unidirectionnels. Un magasin de palettes (composé d'un convoyeur à 
accumulation) permet le stockage des palettes inutilisées. Le magasin comme chacun des postes de travail est une entité indépendante, dans le sens où elle est dotée de sa partie commande, décrite par un GRAFCET indépendant implanté dans l'un des cinq centres de décision.

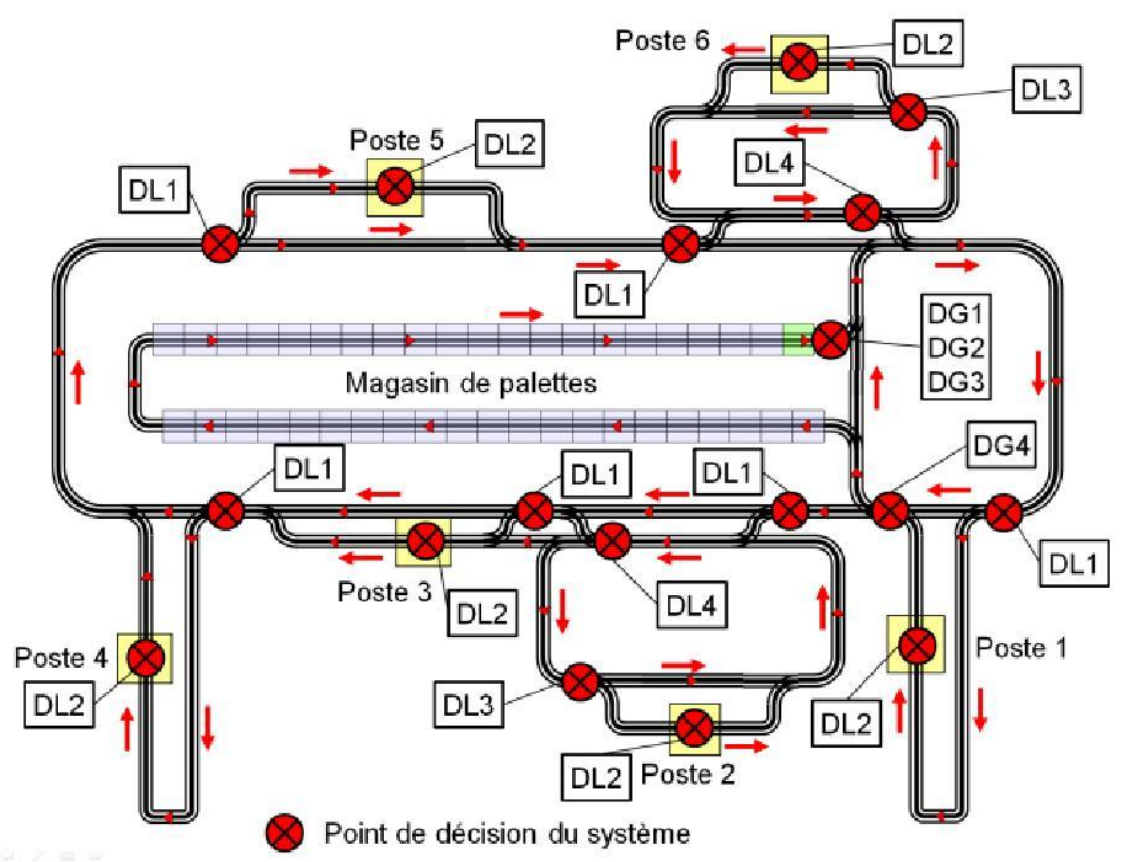

Figure 7. La ligne d'assemblage, avec les points de décision

Le pilotage fait largement appel au concept de pilotage par le produit et se situe au niveau d'intelligence 1 de la classification proposée par Wong et al. (Wong et al. 02). Nous parlerons donc de système informé par le produit. Les palettes sont ici utilisées comme bases pour le transport et la communication des produits avec le système. De ce fait, la production d'un ordre de fabrication (OF) commence par l'union d'un produit avec une base en un "produit informant». Cette union s'accompagne d'un échange d'informations entre le produit et sa base, comprenant un transfert partiel des informations relatives au produit à l'étiquette électronique disposée sur la base. Ces informations concernent la gamme du produit. En fin de production, les données du produit sont mises à jour pour permettre la traçabilité de la fabrication de celui-ci. Parallèlement, la base est réutilisée pour la fabrication du produit suivant, jusqu'au solde de l'OF où la palette retourne au magasin. Pour que cela soit possible, l'étiquette contient également des données relatives à la vie de la palette, indépendamment des produits qu'elle transporte. 
Le produit pilotant se déplace ensuite sur la boucle centrale afin de trouver un poste de travail capable de réaliser la prochaine opération de la gamme du produit et à même de l'accueillir (en termes de capacité, de panne, etc.). Cette négociation est initiée par le produit à son arrivée à l'entrée du poste. C'est une négociation heuristique sans contre-proposition (Nfaoui et al., 2008). La palette indique l'opération à réaliser. Le poste indique, s'il sait faire l'opération, dans combien de temps il pourra traiter la palette. La décision est ensuite prise de prendre ou non la palette. La commande du poste considère à la fois les informations qui lui sont relatives dans la base de données du MES, les données relatives au produit dans l'étiquette électronique et la règle implantée dans la commande pour prendre la décision de laisser rentrer le produit ou non dans le poste.

Ainsi, c'est le produit pilotant qui provoque les décisions le concernant durant son évolution sur la chaine. On ne décide pas au préalable quel poste réalisera une opération, ni quand cette opération sera réalisée. C'est le cheminement du produit sur la ligne qui induira la prise de ces décisions par les entités constituant le système. Lors du lancement d'un OF, l'entité " magasin » doit prendre trois décisions :

- DG1 : À quelle date sera lancé l'OF ?

- DG2 : À combien de produits de l'OF seront affectées chacune des palettes?

- DG3 : Quelle priorité sera donnée à l'OF ?

Cette entité a de plus une décision à prendre en cours de production :

- DG4 : La palette se trouvant à l'entrée du magasin doit-elle y revenir ou doit-elle rester sur la boucle centrale?

Lors du cheminement de la palette, les entités " poste » devront eux même prendre deux décisions :

- DL1 : Le poste doit-il prendre le produit qui se présente à l'entrée ?

- DL2 : En fin d'opération, le produit doit-il passer à l'opération suivante ou doit-il subir une opération additionnelle de retouche?

Les stocks en amont des postes 1,3 , 4, et 5 ont une gestion qui est nécessairement en FIFO. Par contre, les stocks des postes 2 et 6 peuvent avoir une règle de gestion quelconque. Ainsi, ces derniers devront prendre deux décisions supplémentaires:

- DL3 : Le poste doit-il faire entrer dans la zone de travail le produit qui se présente ou doit-il le laisser dans le stock tampon?

- DL4 : Le produit se trouvant dans le stock tampon doit-il y rester ou aller vers la boucle centrale? 
Utilisation d'un observateur pour le pilotage des systèmes contrôlés par le produit 19

\subsection{Aide au pilotage par la simulation}

Parmi l'ensemble de ces décisions, nous avons identifié que deux d'entre elles se prêtaient particulièrement bien à l'aide de la simulation, tout en se différenciant dans les mécanismes mis en jeu.

\subsubsection{Décision globale}

Il s'agit tout d'abord de la décision DG2. Cette décision est prise par un opérateur humain, que nous appellerons pilote de la ligne. Celui-ci doit, lors du lancement d'un nouvel OF au niveau du MES, définir le nombre de produits qui seront associés à chaque palette, ce dont découle le nombre de palettes qui seront affectées à cet $\mathrm{OF}$. Ceci aura une grande influence sur la durée de production totale de l'ordre. Par exemple, pour produire 40 produits, la question est de savoir s'il est plus pertinent d'allouer 1, 20 ou même 40 palettes à l'ordre? Un nombre faible tend à augmenter la durée de production de l'ordre considéré, mais a une faible influence sur les autres ordres en cours sur le système, tandis qu'un nombre élevé permet d'augmenter la parallélisation des opérations, mais tend à surcharger le système, d'autant que le nombre total de palettes sur le système est limité (42).

Cette décision est dite globale car elle nécessite des indications sur le comportement global de la ligne (et notamment des ordres de fabrication la parcourant), et qu'elle influe directement sur l'ensemble du système.

Le but de la simulation en ligne est de prévoir, compte tenu des choix du pilote et de la production en cours, quel seront les dates de fin des OFs en cours et de celui que le pilote cherche à lancer. La connaissance de ces dates de fin d'OF permettra au pilote de faire les meilleurs choix. L'outil est contrôlé via l'écran de supervision du MES. En effet, il est sensé être utilisé par des non-spécialistes de simulation, et un effort particulier doit donc être apporté à l'interface homme-machine. À chaque action sur le bouton "Simulation », les données affichées sur l'écran de supervision et représentant les dates de fin estimées de chaque ordre sont mises à jour avec les nouveaux résultats de simulation. Les ordres concernés peuvent être soit en cours, soit prêts à être lancés. Cette dernière caractéristique permet à l'opérateur d'évaluer la pertinence du paramétrage de ces ordres avant le lancement.

Après que l'opérateur ait initié la demande d'aide à la décision, le modèle de simulation (simulateur) commence par collecter toutes les informations nécessaires à cette simulation, notamment la table des ordres de fabrication ou les paramètres des postes de travail par exemple. Ces données sont situées dans la base de données du MES. Parallèlement, il demande à l'observateur une copie de son état actuel. L'observateur le lui transmet alors sous forme de fichiers texte formatés. À partir du moment où toutes les données lui sont parvenues, le simulateur débute la simulation en s'initialisant sur cet état. Lorsque cette simulation se termine, les résultats sont inscrits dans la base de données du MES, puis un acquittement est envoyé au MES. Celui-ci se charge ensuite de les afficher sur l'écran de supervision. Dans notre 
application, pour des ordres de fabrication ayant une durée d'environ une heure, les résultats sont affichés moins de cinq secondes après que l'opérateur ait cliqué sur le bouton « Simulation ».

Comme on peut le voir, le centre de décision est ici le pilote de la ligne. Celui-ci évalue la pertinence de son paramétrage en fonction des données qui sont affichées sur l'écran de supervision. Ces données contiennent les résultats de la simulation en ligne. La simulation a donc permis d'augmenter l'ensemble $\mathrm{P}$ des paramètres entrant dans la prise de décision. Une direction future, et techniquement envisageable, des travaux sur cette décision est de remplacer le pilote de la ligne par un module d'optimisation entièrement automatisé, qui, à la saisie d'un nouvel OF au niveau de la supervision, calculerait automatiquement le nombre optimal de palettes à associer à l'OF.

\subsubsection{Décision locale}

À l'inverse, la décision DL3 est une décision nécessitant des paramètres provenant d'un sous-ensemble du système (en l'occurrence un poste et son stock), et n'influant directement que sur ce même sous-ensemble. Il est par contre important de remarquer que cette décision aura des conséquences sur le système entier.

Cette décision concerne les postes 2 et 6 . La décision DL3, présentée sur la Figure 8, permet de choisir les produits qui entrent effectivement sur le poste depuis le stock amont. Ce choix est généralement fait à partir d'une règle de gestion de stock ne prenant en compte que l'état actuel du stock.

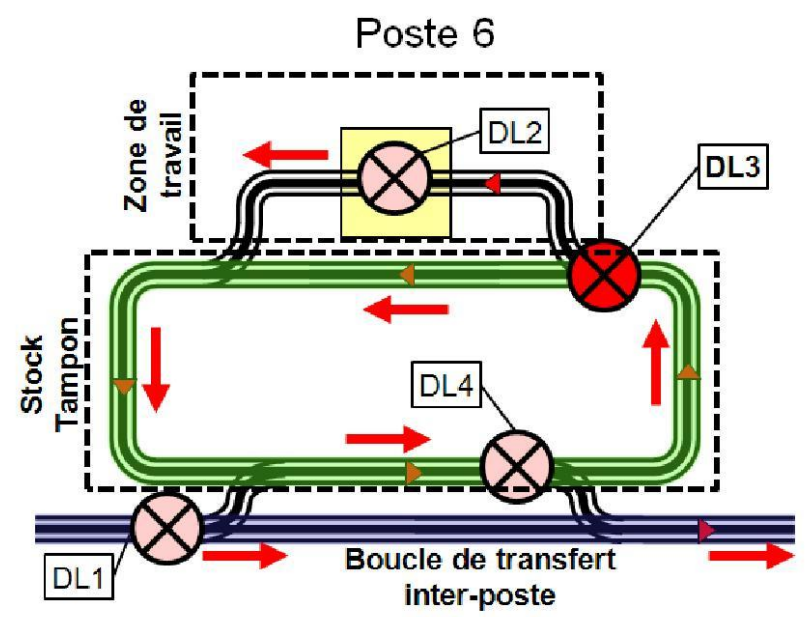

Figure 8. Implantation des postes 2 et 6 : exemple du poste 6 
Le centre de décision associé à DL3 est la commande du poste de travail. Les paramètres pris en considération sont les quantités de chacune des références présentes dans le stock. Le système directement influé par la décision est le poste. Le système influé par la décision est potentiellement l'ensemble du système de production, puisqu'un changement de production sur ce poste peut influer sur la politique de rentrée des produits dans le stock (décision DL4). Il y a ici une opposition entre l'ensemble des paramètres qui ne sont relatifs qu'au $\mathrm{Sd}$, et le $\mathrm{Si}$ qui n'est pas du tout pris en compte. Nous proposons d'utiliser la simulation pour élargir l'ensemble des paramètres à l'ensemble $\{\mathrm{Sd}+\mathrm{Si}\}$ en simulant l'entrée future des produits dans le stock. Ainsi, il sera possible de connaître les quantités futures de chacune des références, et ainsi prendre une décision ayant une probabilité plus faible d'être rapidement caduque.

Cette proposition n'a pour le moment pas été implantée sur le système de production, mais a été testée sur le système émulé. Nous avons montré dans (Cardin, 2006) que l'utilisation de la simulation permettait d'améliorer la règle Clear-afraction, particulièrement performante dans la réduction du nombre de réglages au cours d'une production.

Comme précédemment, la simulation sert ici à élargir l'ensemble $\mathrm{P}$ des paramètres pris en compte dans la décision. Toutefois, la décision est ici déclenchée par le produit, et le centre de décision fait appel automatiquement à la simulation.

\subsection{Implantation matérielle de la solution}

L'architecture de notre système ne comprend pas d'élément du niveau de la planification (pas d'ERP, APS, etc.). Elle se restreint donc, outre les équipements de terrain (niveau de l'opération) et sa commande bas-niveau (niveau de la commande), à un serveur $\mathrm{OPC}$, un observateur et un MES - constitué d'une base de données (SQL Server), d'un utilitaire de supervision et d'un module d'aide à la décision basé sur la simulation (niveau de l'exécution). La Figure 9 présente l'ensemble des éléments de cette architecture avec les moyens de communication en détail. La communication se fait majoritairement sur Ethernet Industriel. Seuls les modules de lecture/écriture des étiquettes électroniques sont connectés aux automates par liaison FipIO et les pupitres par liaison série.

Des travaux antérieurs (Cardin MOSIM 08) nous ont permis de montrer que le temps $\mathrm{R}(\mathrm{ms})$ de réponse lors de la détection d'un évènement sur le réseau de terrain au travers de notre serveur OPC était une fonction linéaire de la période de rafraichissement $\mathrm{p}(\mathrm{ms})$ du serveur de la forme :

$$
\mathrm{R}=1.27 \mathrm{p}+80
$$

Une période de rafraichissement de $50 \mathrm{~ms}$ a été choisie pour notre installation, ce qui correspond à un temps de réponse inférieur à $150 \mathrm{~ms}$. Relativement à la 
dynamique de notre système, ceci correspond à un déplacement de 3 centimètres. Cette donnée nous permet de justifier l'utilisation d'une telle architecture matérielle dans une classe de systèmes ayant une dynamique comparable.

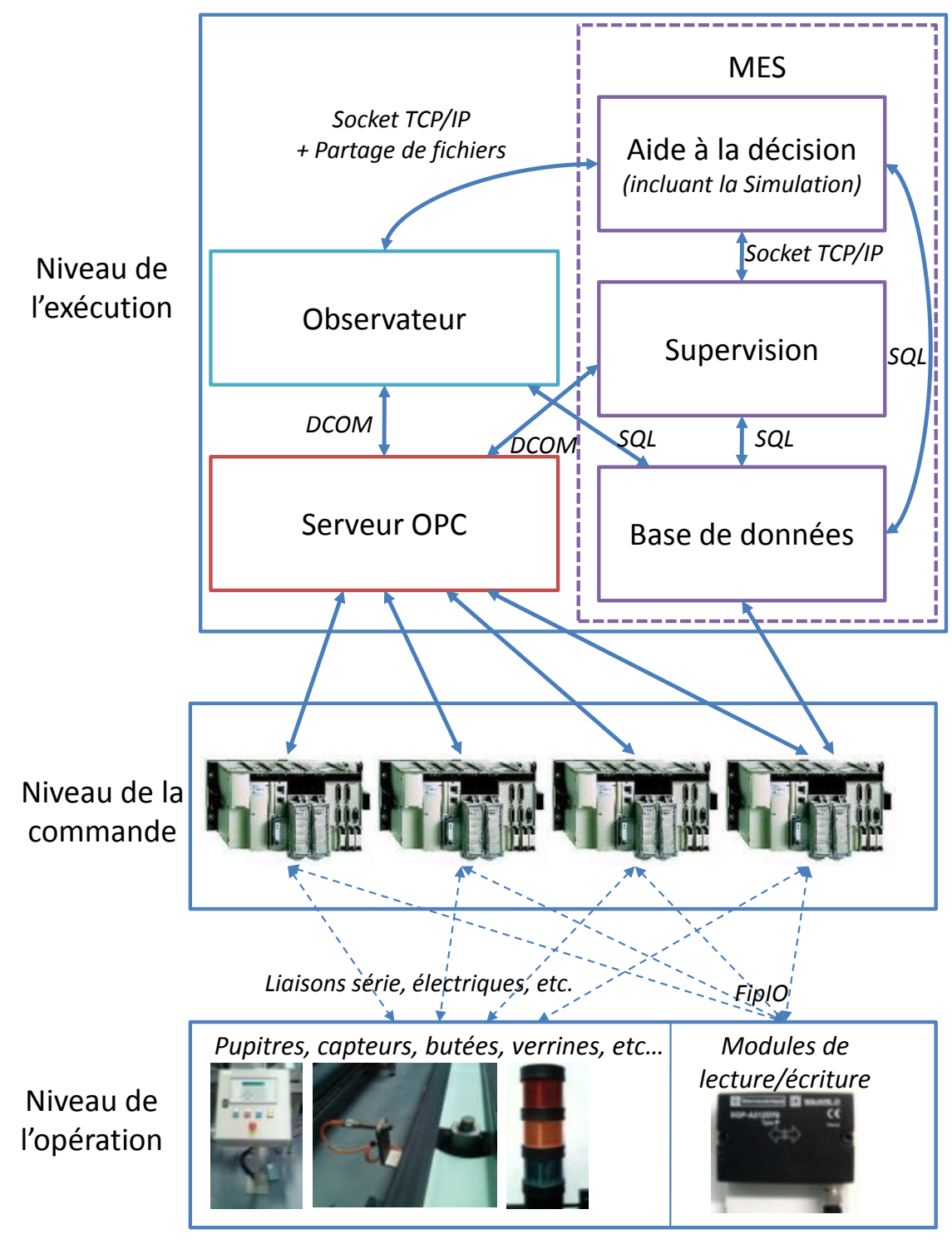

Figure 9. Architecture matérielle complète de la solution implantée (Cardin, 2007) 


\section{Conclusion et perspectives.}

Après avoir rappelé le principe du pilotage par le produit, nous avons montré comment la simulation pouvait devenir un outil important d'aide au pilotage des systèmes contrôlés par le produit. Cet outil est destiné à aider chacun des centres de décision locaux à prendre des décisions en prenant en compte le comportement global du système de production. De ce fait, ce papier est à la fois destiné à présenter un outil d'aide à la vision, permettant à chaque centre de décision d'avoir une vue globale sur l'état du système à tout instant, pour alimenter sa réflexion, et un outil d'aide à la prévision, lui permettant de prévoir les conséquences des alternatives qu'il a formulées sur le comportement futur du système.

De part sa démarche expérimentale, la simulation peut se révéler relativement longue, sa durée étant incompatible avec une prise de décision en temps réel. L'une des pistes de solution est d'adjoindre à la simulation une couche d'optimisation. Nous réalisons actuellement une plateforme expérimentale joignant simulation en ligne et optimisation, dans le cadre d'une application industrielle. Une autre piste et d'utiliser d'autre outils de prévision que la simulation, cette dernière étant gardée pour assurer l'observation du système.

Le concept de pilotage par le produit ouvre des perspectives nouvelles pour aller vers des systèmes de production agiles et réactifs. Mais il nous semble que de la qualité des décisions prises dans ces système dépendront les améliorations apportées par les SCP.

\section{Références}

Cardin O., Apport de la simulation en ligne dans l'aide à la décision pour le pilotage, Thèse de doctorat, Université de Nantes, 2007

Cardin O., Castagna P., «Handling uncertainty in production activity control», Proceedings of the 12th IFAC Symposium on Information Control Problems in Manufacturing, INCOM 2006 , Saint-Etienne, France, p. 579-584, 2006.

Cardin O., Castagna P., Chové E., «La simulation pour l'aide au pilotage des systemes controlés par le produit », MOSIM'08, Paris, France, 2008.

Castagna P., Mebarki N., Gauduel R., «La simulation, un outil d'aide au pilotage des systèmes de production. Exemples d'application. » MOSIM'01, Troyes, France, 2001.

Cea A., Bajic E., « Ambient Services for Smart Objects in the Supply Chain Based on RFID and UPnP Technology ", Proceedings of the Third Conference on Management and Control of Production and Logistics MCPL'04. Santiago de Chile, 2004. 
Davis, W. J., « Online simulation: Need and evolving research requirements », dans Banks J. Handbook of simulation, p. 465-516, John Wiley and Sons, New-York, 1998.

Drake, G. R., Smith J. S., « Simulation system for real-time planning, scheduling, and control. » 28th conference on Winter simulation. Coronado: ACM Press, 1996. pp. 1083 - 1090.

Gouyon D., Pétin J.-F., Morel G., «A product driven reconfigurable control for shop floor systems », Studies in Informatics and Control, vol. 16, 2007.

McFarlane D., Carr J., Harrison M., McDonald A., « Auto-ID's three R's: Rules and Recipes for product Requirements », Auto-ID Centre White Paper, 2002.

Nfaoui H., Ouzrout Y., Bouras A., El Beqqali O., «Architecture distribuée à base d'agents pour la simulation proactive et l'aide à la décision dans la chaine logistique », MOSIM'08, Paris, France, 2008.

Pannequin R., Proposition d'un environnement de modélisation et de test d'architectures de pilotage par le produit de systèmes de production, Thèse de doctorat, Université Henri Poincaré de Nancy, 2007

Pétin J.-F., Gouyon D., Morel G., « Supervisory synthesis for product-driven automation and its application to a flexible assembly cell », Control Engineering Practice, vol. 15, n5, 2007, p. 595-614.

Pinot G., Cardin O., Mebarki N., «A study on the group sequencing method in regards with transportation in an industrial FMS », IEEE International Conference on Systems, Man and Cybernetics, Montréal, Québec, 2007.

Pujo P., Kieffer J.P., « Chapitre 1: Concepts fondamentaux du pilotage des systèmes de production », dans Pujo P., Kieffer J.P. Fondements du pilotage des systèmes de production, Traité IC2 Productique, Edition Hermès, 2002.

Rogers P., Flanagan M.T., «On-line simulation for real-time scheduling of manufacturing systems », Industrial Engineering, vol. 23, 1991, p. 37-40.

Roy B., « Main sources of inaccurate determination, uncertainty and imprecision in decision models », Mathematical Computer Modeling, vol. 12, $\mathrm{n}^{\circ}$ 10/11, p. $1245-1254,1989$.

Trentesaux D., Pilotage hétérarchique des systèmes de production, Habilitation à Diriger des Recherches, Université de Valenciennes et du Hainaut-Cambresis, 2002.

Valckenaers, P., Van Brussel, H., Wyns, J., Bongaerts, L., Peeters, P., « Designing Holonic manufacturing systems", Robotics and Computer-Integrated Manufacturing, vol. 14, p. 455-464, 1998. 
Utilisation d'un observateur pour le pilotage des systèmes contrôlés par le produit 25

Valckenaers, P., Karuna, H., Saint Germain, B., Verstraete, P., Van Brussel, H.. «Emergent short-term forecasting through ant colony engineering in coordination and control systems », Advanced Engineering Informatics, vol.20, n³, p.261-278, 2006.

Van Brussel, H., Wyns, J., Valckenaers, P., Bongaerts, L., Peeters, P., « Reference architecture for holonic manufacturing systems: PROSA », Computers in Industry, vol. 37, p. 255-274, 1998.

Wong, C. Y., McFarlane D., Zaharudin A. A., Agarwal V., « The Intelligent Product Driven Supply Chain », IEEE Systems Man and Cybernetics, Hammammet, Tunisie, 2002. 